\title{
Community assembly in plant-bacterium symbiotic interactions in a natural environment: Does host genetic variation matter?
}

\author{
Shinnosuke Kagiya ${ }^{1}$, Ken-ichi Kucho ${ }^{2}$, and Shunsuke Utsumi ${ }^{3}$ \\ ${ }^{1}$ University of the Ryukyus \\ ${ }^{2}$ Kagoshima University \\ ${ }^{3}$ Hokkaido University
}

September 24, 2021

\begin{abstract}
Community assembly of diverse mutualistic symbionts in host plants have recently received much attention. On the other hand, for decades, researchers have also focused on a role of genetic variation for shaping ecological communities. However, it remains unclear how local interaction networks between a host and symbionts are shaped from the pool of genetically diverse microorganisms in the natural rhizosphere. In this study, we comprehensively analyzed local and regional genetic communities of Frankia in host individuals and in surrounding soils using metabarcoding to unravel community assembly in shaping interaction networks between plants and root nodule symbionts, both of which are genetically diverse, in natural ecosystems. We found that Frankia communities in rhizosphere soil were consisted of tremendously diverse strains. The diverse Frankia communities in rhizosphere soils were heterogeneously structured by the presence of host individuals and soil nutritional status. Furthermore, quantification of the filtering forces by community analysis demonstrated that the filtering forces that shape symbiotic networks would depend on genetic variation in the host plant. This study suggests that filtering mechanisms and host genetics would have a profound role in shaping plant-bacterium symbiotic networks in natural environments.
\end{abstract}

Title: Community assembly in plant-bacterium symbiotic interactions in a natural environment: Does host genetic variation matter?

Authors: Shinnosuke Kagiya ${ }^{1}$, Ken-ichi Kucho ${ }^{2}$, Shunsuke Utsumi ${ }^{3 *}$

Affiliations:

${ }^{1}$ Graduate School of Environmental Science, Hokkaido University, North 10, West 5, Sapporo, Hokkaido 060-0810, Japan

${ }^{2}$ Graduate School of Science and Engineering, Kagoshima University, Korimoto 1-21-35, Kagoshima 8900065, Japan

${ }^{3}$ Field Science Center of Northern Biosphere, Hokkaido University, North 9, West 9, Sapporo, Hokkaido 060-0809, Japan

${ }^{*}$ Corresponding author

Shunsuke Utsumi

Field Science Center of Northern Biosphere, Hokkaido University, North 9, West 9, Sapporo, Hokkaido 060-0809, Japan

e-mail: utsumi@fsc.hokudai.ac.jp 


\begin{abstract}
Community assembly of diverse mutualistic symbionts in host plants have recently received much attention. On the other hand, for decades, researchers have also focused on a role of genetic variation for shaping ecological communities. However, it remains unclear how local interaction networks between a host and symbionts are shaped from the pool of genetically diverse microorganisms in the natural rhizosphere. In this study, we comprehensively analyzed local and regional genetic communities of Frankia in host individuals and in surrounding soils using metabarcoding to unravel community assembly in shaping interaction networks between plants and root nodule symbionts, both of which are genetically diverse, in natural ecosystems. We found thatFrankia communities in rhizosphere soil were consisted of tremendously diverse strains. The diverse Frankia communities in rhizosphere soils were heterogeneously structured by the presence of host individuals and soil nutritional status. Furthermore, quantification of the filtering forces by community analysis demonstrated that the filtering forces that shape symbiotic networks would depend on genetic variation in the host plant. This study suggests that filtering mechanisms and host genetics would have a profound role in shaping plant-bacterium symbiotic networks in natural environments.
\end{abstract}

Key words

Alnus hirsuta, Frankia, genetic variation, metabarcoding, nif D-K IGS region, nitrogen-fixing bacteria, root nodule symbiosis

\title{
Introduction
}

Mutualistic interactions with microsymbionts are ubiquitous in the roots of almost all terrestrial plants. Patterns and processes of community assembly of diverse mutualistic symbionts in host plants have begun to receive great attention along with the recent advances in high-throughput molecular barcoding techniques (Vályi et al., 2016; Bitterbiere et al., 2020). It has been increasingly reported how local symbiont communities assemble through abiotic and biotic filters from a regional pool, especially for mycorrhizal fungi (de Souza \& Freitas, 2017; Lekberg et al., 2011; López-García et al., 2017; Bitterbiere et al., 2020). On the other hand, for decades, researchers have also demonstrated significant effects of genetic variation within a species on shaping ecological communities (Bailey et al., 2009; Crutsinger et al., 2014; Kagiya et al., 2018; Whitham et al., 2006). In plant-microsymbiont interactions, many previous studies have demonstrated that mutualistic interactions with microbial symbionts increase or decrease the performance of host plants, depending on genetic variation in infected symbionts, including the growth, survival, reproduction, and defense (Ballhorn et al. 2017; Barrett et al, 2012; 2015; Broughton et al., 2003; Clawson et al., 1998; 1999; Heath \& Stinchcombe, 2014; Mensah et al., 2015). Nevertheless, it remains unclear how local interaction networks between a host and symbionts are shaped from the pool of genetically diverse microorganisms in the natural rhizosphere.

Genetic variation in a host plant population may also provide great insights for ecological and evolutionary perspectives in microsymbiont assembly in nature. This is because genetic variation within a plant species is generally known for one of primary factors that shape the assemblages of soil microbiomes in terms of species richness and abundance (Evans et al., 2016; Genung et al., 2013; Lamit et al., 2015; Lankau, 2011; Schweitzer et al., 2008). However, the influences of genetic variation in host plants on the assembly of interacting microsymbionts remain unclear. Because inefficient variants necessarily prosper and break down the reciprocal mutualism, there are filtering mechanisms for the reciprocal mutualism between hosts and symbionts (Archetti et al., 2011; Heath \& Stinchcombe, 2014). Several mechanisms are involved in the stabilization of mutualistic partnerships in environments with lower quality partners; for example, partner choice, in which host plants can choose the optimal partner based on preinfection signals (Kiers \& Denison, 2008), and sanctions, in which host plants monitor the mutualistic benefits from bacteria and punish less nitrogen-fixing nodules to favor cooperation nodules (Denison, 2000; Kiers \& Denison, 2008; Kiers et al., 2003; 2007; Materon \& Zibilske, 2001; West et al., 2002b). Given that plants are ubiquitously exposed to genetically diverse microsymbionts in nature, including both efficient and inefficient variants, the maintenance of plant-microbe mutualistic symbiosis in the field should be achieved through these filtering mechanisms. The filtering processes from the microbial community pool into host roots are important for the understanding of 
network structure, community dynamics, and dispersal of microbial symbionts in natural ecosystems (Valyi et al., 2016). However, because theoretical and experimental approaches have been primarily applied, to our knowledge, no studies have successfully demonstrated the filtering activities and/or their outcomes in genetic associations between plants and microsymbiont mutualists in natural ecosystems.

To address host plants' filtering specific symbiont strains into their roots from surrounding microsymbiont communities in nature, we need to distinguish between infecting and non-infecting microsymbiont communities. The mutualism between the nitrogen-fixing bacteriaFrankia sp. (Frankiaceae) and the alder tree (Alnus ), known as "Frankia-Alnus mutualism," provides a good opportunity to study the communities of both co-infecting symbionts and non-infecting relatives in nature. Frankia form nodules on actinorhizal host plants, such as Alnus, Myrica and Casuarina . Because Frankia bacteria are also free-living in soil (Benson \& Dawson, 2007), this root nodule symbiosis is likely to be effective to successfully distinguish communities of symbionts infecting hosts and communities of close relatives that are present in the soil around hosts but are non-infecting.

The goal of this study is to elucidate the filtering effects of a host plant on associated microsymbiont communities in a natural condition. We aim to (1) comprehensively investigate local and regional AlnusFrankia symbiotic communities in host root nodules and rhizosphere soils in the field, using next-generation sequencing (NGS); (2) compare the genotypic composition of Frankia bacteria between nodules and soils to examine the consequences of filtering forces; (3) examine how abiotic environmental factors and genetic variation in hosts affect Frankia communities, and (4) examine how host-assembled symbionts are associated with host genetic variation.

\section{Materials and Methods}

\section{Host species and nitrogen-fixing bacteria}

Alnus hirsuta (Betulaceae; Alnus incana ssp.hirsuta Spach; Chen \& Li 2004; Ren et al. 2010) is a deciduous, broad-leafed tree and an early successional species. It is widely distributed in temperate riparian forests in Japan, northeastern China, Korea, and Russia. Alnus trees have characteristics as a foundation species (Ellison et al., 2005; 2010; Kagiya et al., 2018), which is likely to have great impacts on forest ecosystems.

Frankia sp. (Frankiaceae) are actinobacteria with the ability to convert atmospheric nitrogen gas to ammonia. While Frankia that form nodules on roots of phylogenetically diverse host plants are gram-positive, rhizobia that form nodules on roots of legume plants are gram-negative.

Study sites

Our study sites are located in and around Uryu Experimental Forest, Japan (44 030-290N, $\left.142^{\circ} 010-200 \mathrm{E}\right)$, a natural conifer-broadleaf mixed forest. Sampling of rhizosphere and riparian soils and root nodules from alders was conducted in 12 sites along four river areas (see also Kagiya et al. 2018). Only Alnus hirsuta exist in these sites actinorhizal plants.

Sampling of root nodules, rhizosphere soils, and riparian soils

In the study sites, we collected root nodules, rhizosphere soils (i.e., inside under host tree crown), and riparian soils (i.e., riparian but outside of alder crown). Root nodules were collected from the roots of 78 adult trees, whose genetic variation was estimated using 1,077 single nucleotide polymorphism (SNP) markers (Kagiya et al., 2018). A nodule cluster was collected from each of seven roots per alder individuals. The sampling was performed in August 2017.

Rhizosphere soils were collected between August and September 2018. Following litter layer removal, we collected three $10-\mathrm{cm}^{3}$ soil blocks from the inside area under each of the alder crowns using a sterilized shovel. The shovels were sterilized at $121{ }^{\circ} \mathrm{C}$ for $20 \mathrm{~min}$ before using for the sampling, and disinfected using $70 \%(\mathrm{v} / \mathrm{v})$ ethanol after every soil sampling event. We changed shovels to new ones when we moved to other sites. 
To sample riparian soils, we established 40-m transects along the rivers at the centroid points of the studied alder individuals within each of the study sites. The riparian soils samplings were conducted at every 10 $m$ along the transects (i.e., four points in total per each site). At the points, we collected $10-\mathrm{cm}^{3}$ soil blocks in the same way of rhizosphere soil samplings. The riparian soils samplings were performed from July to August in 2017. From these samplings, we obtained a total of 12 riparian soil samples. To investigate environmental conditions, at the sampling points, soil $\mathrm{pH}$ was measured using a direct soil $\mathrm{pH}$ probe kit (Hanna Instruments). After transporting the soils to the laboratory, total inorganic nitrogen, including $\mathrm{NH}_{4}{ }^{+}$ and $\mathrm{NO}_{3}{ }^{-}$, was extracted from $10 \mathrm{~g}$ of the sampled riparian soils by $100 \mathrm{~mL}$ of $2 \mathrm{M} \mathrm{KCl}$, and analyzed with an auto analyzer (AACS-4, BL-TEC Inc., Japan; the detailed method of total inorganic nitrogen analysis was previously described by Fukuzawa et al., 2006).

Frankia DNA extraction from root nodules and soils

The collected nodule and soil samples were transported to a laboratory in a cool box, and then we immediately extracted Frankia DNA. The root nodules were sterilized using 10\% (v/v) Clorox bleach. One lobe was picked from each washed nodule, sliced using sterilized razor blades, and crushed using sterilized homogenization sticks. Bacterial DNA extraction from the crushed lobes and $300 \mathrm{mg}$ of the soil samples from alder rhizosphere and riparian soils was performed using a DNeasy Blood \& Tissue Kit (Qiagen) and NucleoSpin Soil (Macherey-Nagel), respectively.

\section{Preparation of library for NGS}

For the rhizosphere and riparian soil samples, a nested polymerase chain reaction (PCR) was performed to amplify a nif D-K intergenic spacer (IGS) region in the samples. We adopted nested PCR to collect a sufficient amount of Frankia DNA for NGS (Ben Tekaya et al., 2018; Rodriguez et al., 2016). An approximately 500-bp fragment of thenif D-K IGS region was amplified by the PCR approach with theFrankia -specific primer pair, nif D1310frGC and nifKR331frGC (Kagiya and Utsumi, 2020). PCR amplification was performed as follows: one cycle at $95{ }^{\circ} \mathrm{C}$ for $2 \mathrm{~min}$, followed by 35 cycles at $95{ }^{\circ} \mathrm{C}$ for $1 \mathrm{~min}$ and $64{ }^{\circ} \mathrm{C}$ for $5 \mathrm{~min}$, and a final step of one cycle at $72{ }^{\circ} \mathrm{C}$ for 5 min. The PCR products of soil samples and extracted DNA from host nodules were subjected to the same PCR method. PCR was performed to amplify an approximately 350-bp fragment of the nif D-K IGS region with the Frankia -specific primer pair (Table S1). This process was performed using the same method as the first-stage PCR for the usual NGS library preparation workflow. PCR amplification was performed as follows: one cycle at $95{ }^{\circ} \mathrm{C}$ for $2 \mathrm{~min}$, followed by 35 cycles of $95{ }^{\circ} \mathrm{C}$ for $1 \mathrm{~min}$ and $61{ }^{\circ} \mathrm{C}$ for $5 \mathrm{~min}$, and a final step of one cycle at $72{ }^{\circ} \mathrm{C}$ for $5 \mathrm{~min}$. All successful PCR products were cleaned using AMPure XP magnetic beads (Beckman Coulter). These cleaned products were tagged by unique indices per sample performing index. Index PCR was performed as follows: one cycle at $95{ }^{\circ} \mathrm{C}$ for 3 min, followed by 8 cycles at $95{ }^{\circ} \mathrm{C}$ for $30 \mathrm{sec}, 55{ }^{\circ} \mathrm{C}$ for $30 \mathrm{sec}$ and $72{ }^{\circ} \mathrm{C}$ for $30 \mathrm{sec}$, and a final step of one cycle at $72{ }^{\circ} \mathrm{C}$ for 5 min. After the index PCR, the products were cleaned again with AMPure magnetic beads, and then libraries with the same amplicon concentration were pooled. These samples were analyzed, using Illumina MiSeq with paired-end 300 bp reads (Macrogen, Korea).

\section{Bioinformatics}

Obtained paired-end reads were filtered and processed by the DADA2 pipeline (Callahan et al., 2016) in QIIME2-2019.01 (Bolyen et al., 2019). Because the soil samples were amplified via nested PCR, sequences representing $<1 \%$ of the total number of reads per sample were removed using the resulting BIOM table and a customized R script (Ben Tekaya et al., 2018; Rodriguez et al., 2016). For nodule samples, the samples that included numbers of reads within 99 percentiles of the total number of all sample reads were used in the subsequent analyses $(n=72)$. Then, we obtained amplicon sequence variants (ASVs). The sequences were interrogated with BLAST+2.9.0 BLASTN (Camacho et al., 2009) to remove non-Frankia sequences. These sequences were then clustered via operational taxonomic unit (OTU) separation, using the sequences of 18 uncultured Frankia strains as references (i.e., OTU01-18 strains; accession number: LC482655-LC482672; Kagiya and Utsumi 2020). OTU separation was performed using the CD-Hit program (Li \& Godzik, 2006) at $97.0 \%$ similarity threshold. This threshold was decided based on Põlme et al. (2014) and Kagiya and 
Utsumi (2020).

Phylogenetic trees (Fig. S2) were constructed using maximum likelihood (ML; bootstrap analyses with 1000 replications) based on the Kimura two-parameter evolutionary model (Kimura, 1980) with a discrete gamma distribution, which was selected by the evolutionary model selection procedure in MEGA 7.0.21 (Kumar et al., 2016). ML phylogenetic trees were generated in MEGA 7.0.21 (the detailed method of ML phylogeny was previously described by Kagiya et al. 2020).

\section{Community analysis}

The community data of Frankia OTUs was standardized by coverage-based rarefaction (Chao \& Jost, 2012) and transformed to presence/absence data. Non-metric multidimensional scaling (NMDS) was performed to visualize Frankia community dissimilarity in this study.

To elucidate whether filtering force drives symbiont assembly into host plants, we generated 1000 null models of symbiont community by seven random samplings from overall rhizosphere source community with frequency distribution of OTUs in rhizosphere soil. We comparedFrankia OTU richness and composition in host's root-nodules with the null model, using generalized linear model (GLM) with Poisson distribution and permutation MANOVA (PERMANOVA) with 9999 permutations, using the lme4 1.1-21 package (Bates et al., 2019) and the vegan 2.5-5 package of the R 3.6.1 software, respectively.

To test how abiotic environmental factors and genetic variation in hosts affect Frankia communities, we analyzed correlations ofFrankia community dissimilarity with Nei's genetic distance of hosts, soil $\mathrm{pH}$ and inorganic nitrogen contents in soil, using the vegan 2.5-5 package in the $\mathrm{R}$ 3.6.1 software.

To quantify variation in symbiont filtering force among hosts, we defined the "symbiosis filtering" index as dissimilarity inFrankia community composition between root nodules and rhizosphere soils in each river area. This symbiosis filtering index indicates how certain Frankia genotypes were filtered from a genetic pool in each river area. In order to quantify differences in symbiosis filtering index, we calculated the symbiosis filtering indices as Jaccard dissimilarities between root-nodules of host individuals and rhizosphere soils in each river area, using 56 individuals of studied alders which had Frankia community data for both the root nodules and rhizosphere soils. Then, the symbiosis filtering indices were averaged for each site. Next, we calculated the Euclidian distance of the averaged symbiosis filtering indices among sites. We referred to the distance as "symbiosis filtering differences." In addition, to estimate the genetic distance of alder populations among sites, we calculated Nei's genetic distance (Nei, 1978), based on 1,077 SNPs that had been obtained by RAD-Seq (Kagiya et al., 2018). Finally, the correlation between the Nei's genetic distance of alders and the symbiosis filtering differences was examined using Mantel tests. All the community analyses were performed with the vegan $2.5-5$ package in the $\mathrm{R}$ software (version 3.6.1).

To quantify the relative contribution of genetic distance of hosts and other abiotic factors in explaining the spatial patterns of symbiosis filtering, accounting for spatial autocorrelation, generalized dissimilarity modeling (GDM; Blois et al., 2013; Capinha et al., 2015; Ferrier et al., 2007; Lasram et al., 2015) was performed. GDM models for the symbiosis filtering differences included Nei's genetic distance of hosts, soil pH, and the total amount of inorganic nitrogen in soils, Jaccard community dissimilarity of Frankia in rhizosphere soils, and spatial distance as predictors. To test whether the genetic distance of alders could explain the symbiosis filtering differences without other factors, we estimated the unique and shared contribution of each predictor to the total deviance explained by partitioning the predictors with GDM models (Capinha et al., 2015; Fitzpatrick et al., 2013; Kagiya et al., 2018). To calculate the unique contribution of genetic distance and other factors, the deviance explained of the model removed each predictor was subtracted from the deviance explained of the full model. To calculate the shared contributions of both predictors, a value of deviance explained of the model that was fitted by both predictors was subtracted from the unique contributions of each predictor.

\section{Results}

Genetic composition of symbiotic and free-living Frankia communities in Alnus-Frankia networks 
We obtained a total of 352 ASVs from 138 samples consisting of riparian soil, rhizosphere soil, and root nodules (Fig. S1). Of the total ASVs, $90.34 \%$ were unique singleton variants, which occurred once in 138 samples. We found profoundly diverse ASVs from rhizosphere and riparian soils (rhizosphere: 228 ASVs, $n=54$; riparian: 103 ASVs, $n=12$ ). In contrast, in root nodules, the total number of ASVs was quite limited ( 38 ASVs, $n=72$ ). These results suggest that host individuals establish symbiosis only with limited Frankiagenotypes in soil.

To classify the Frankia genetic variants, we used OTU methods based on the genetic similarity of the nif D-K loci. A total of 19 OTUs were obtained at a 97\% similarity threshold. Consequently, most ASVs from root nodules and riparian soils converged to three OTUs (OTU01, 02, and 03), while a variety of OTUs were found in the rhizosphere soil (Fig. 2). Consistently, the sample-based rarefaction curves showed that the number of Frankia OTUs did not completely reach saturation but were significantly greater in number in rhizospheres than in root nodules after standardization of the sample size (Fig. S3). In addition, the number of Frankia OTUs was lower in riparian soils than in rhizosphere soils (Fig. S3). These findings suggest that the occurrence of host plants promote the local assembly of nitrogen-fixing bacterial symbionts in soil rather than homogeneity dispersion of diverse bacterial strains in a natural forest.

Moreover, the phylogenetic trees indicated that the Frankia OTUs detected in our study covered a wide variety of Alnus -infecting strains (Fig. S2). The most abundant strains (i.e., OTU01, 02, and 03) belonged to phylogenetically distant clades (Fig. S2), indicating that the Alnus host population accepts genetically diverse bacterial symbionts.

\section{Filtering force for Frankia assembly on host root-nodules}

The Frankia composition in the root-nodule community was significantly different from that in the rhizosphere community (PERMANOVA: $P<0.001$ ). Community variance ofFrankia in rhizosphere soils was greater than that in host root-nodules (PERMDISP: $P<0.001$; Fig. 2a). The communities of Frankia in host root nodules consisted of a small proportion of the overall members of the communities in the rhizosphere soils (8/16 OTUs were detected from both nodules and rhizosphere soils). Furthermore, rank-abundance dominance plots showed that three OTUs (OTU01, 02, and 03) were dominant and abundance decay was very steep in root nodules (Fig. S4). However, dominance was not obvious and abundance decay was gradual in rhizosphere communities (Fig. S4). These results show that a host plant establishes a symbiotic interaction with limited members from a diverse resource of symbionts in soil.

The number of Frankia OTUs from root nodules was significantly lower than the expected OTU richness by random sampling of the rhizosphere composition (Fig. 2b). Moreover, significantly different Frankia composition was found in the root nodules in the null model (PERMANOVA: $P<0.001$ ). These results provide evidence that filtering force works to shape symbiont communities in the host plant with strains in narrower ranges of Frankia strains than expected from Frankia pools in soil.

\section{Effects of abiotic environmental factors and host genetic variation on Frankia assembly}

The dissimilarity of Frankia communities in root nodules was not associated with the genetic distance of hosts $(r=-0.309, P=0.903$; Table 1$)$. Community composition in symbionts is consistent across host individuals despite host genetic variation. Furthermore, the Frankia community dissimilarity in rhizosphere soils was also not associated with the host's genetic distance $(r=-0.137, P=0.754$; Table 1$)$. However, the structure of Frankia communities in rhizosphere soils was significantly affected by inorganic nitrogen in soils $\left(R^{2}=0.581, P=0.017\right.$; Table 1$)$, while there was no significant relationship between soil $\mathrm{pH}$ and Frankia communities $\left(R^{2}=0.212, P=0.371\right.$; Table 1$)$.

\section{Host genetic variation in symbiosis filtering}

Symbiosis filtering differences were significantly correlated with host genetic distance (Fig. 3a). This correlation remained significant even when spatial distance among alder individuals was corrected using a partial Mantel test $(r=0.444, P<0.001)$. Furthermore, GDM analysis quantified the explanatory proportions of the genetic distances of the hosts and other predictors, including spatial distance among hosts, soil $\mathrm{pH}$, 
amount of inorganic nitrogen in soils, and community dissimilarity of Frankia in rhizosphere soils, for symbiosis filtering differences (Fig. 3b). The genetic distance of alders and other factors absolutely explained the $36.64 \%$ deviance in the symbiosis filtering differences.

Where the deviance explained by the model with genetic distance and other factors was $100 \%$, the genetic distance of alders uniquely explained $47.94 \%$ of the deviance while the other factors uniquely explained $1.31 \%$ (Fig. 3b). This result demonstrates that the host genetic distance was the best of the measured predictors for symbiosis filtering differences.

Taken together, our findings suggest that differences in symbiosis filtering force among host individuals could generate slight differences in symbiont composition among host individuals in order to alter members of mutualistic bacteria obtained from rhizosphere soils, while symbiosis filtering might work to preserve high homogeneity of symbiont composition in host individuals. Moreover, the differences in symbiosis filtering force were likely to covary with the genetic variation of host plants rather than the soil environments surrounding the hosts.

\section{Discussion}

This study highlights how Frankia -host symbiotic communities are shaped through spatial Frankia assembly and filtering forces in nature. We comprehensively investigated genetic diversity and composition of Frankia in different environments, using NGS. The occurrence of host plant individuals promoted the local assemblage of diverse Frankia strains and the greater ASV and OTU richness of Frankia detected in rhizosphere soils around hosts compared to root nodules and host-absent riparian soil. In addition, our results suggest that the diverse Frankia community assemblies in rhizosphere soils are affected by soil nitrogen. However, theFrankia composition in root nodules consisted of very few members (Fig. 1). Because Frankia richness in root-nodules was significantly lower than in the null model, our findings indicate that members of symbionts within hosts were selected from surrounding pools via filtering forces. The community dissimilarity approach suggests that genetic variation in host plants underlies the symbiosis filtering difference even though host genetics did not affect Frankiacompositions in nodules or the rhizosphere.

Filtering of mutualistic partnerships in the field

Many theoretical and empirical studies have shown that stabilizing mechanisms maintain mutualistic interactions in environments with a high genetic diversity of mutualistic partners, including cheaters (Archetti \& Scheuring, 2013; Kiers \& Denison, 2008; Kiers et al., 2007, 2003; West et al., 2002ab). However, to our knowledge, this is the first study to reveal host filtering for genetic variants under field conditions through explicit comparison between colonized symbionts and the regional community pool. In our findings, Frankia communities in host individuals were constructed by smaller numbers of Frankia OTUs than the number of Frankia OTUs in the surrounding environments (Fig. S1, 2, S1). Moreover, the comparison with the null models showed that the Frankia communities in the host plant were not chosen from soils by chance (Fig. $2 \mathrm{~b})$. As a result, symbiont communities in nodules were relatively constant across host plant individuals. SeveralFrankia OTUs were detected from both rhizosphere soils and root nodules (e.g., OTU01, 02, 03, 25, 26, and 28; Fig. 1) and, while these OTUs should have infection ability, OTU 25, 26, and 28 infected only a few host individuals. In addition, even though the occurrences of OTU01, 02, and 03 were not frequent in rhizosphere, it was constantly found from nodules of most trees. Thus, the differences in bacterial assembly between roots and rhizospheres would not depend on infective or non-infective strains, but filtering mediated in determining symbiont members from the genetically diverse Frankia pool.

While filtering forces are likely to regulate symbiont assembly into a host plant in a natural system, most alders interacted with phylogenetically different OTUs (i.e., OTU01, 02, and 03; Fig. 1, S1). This may be a result of genetic variation in multiple bacterial functions. Nitrogen fixation is the fundamental function of nodulating bacteria including Frankia bacteria, promoting not only the growth of host plants but also survival and herbivory defense. Dean et al. (2014) found that nitrogen resources from nitrogen-fixing symbionts were needed for induced herbivory resistance and artificial fertilizer did not induce the resistance. This suggests that herbivore resistance does not depend on the amount of total nitrogen supply but on specific nitrogen 
forms, such as amides and ureides, provided by nitrogen-fixing symbionts. Nitrogen fixed by associated rhizobacteria, includingFrankia, can be stored in nodules and transported to aerial parts as these specific forms (Berry et al., 2011; Dean et al, 2009; Schubert, 1986). Furthermore, Frankia bacteria have some other plant growth promoting functions, such as solubilization of inorganic phosphate (Sayed et al., 2002) and the synthesis of plant hormones (Peret et al., 2007) and siderophores (Tisa et al., 2016). Nouioui et al. (2019) discovered genes encoding these functions from some Frankiastrains in their in silico genome analyses and detected variation in the genes among the Frankia strains. The symbioses with genetically diverse microsymbionts could be explained by the complimentary effects of genetic variation in these multiple functions of Frankia strains on host performance.

In addition, some of the Frankia strains that were obtained in a previous study, which focused on host seedlings (Kagiya and Utsumi, 2020), were not detected in the root nodule samples of most host trees in this study (Fig. S1). This may be explained by the following: (1) the above strains might be extremely rare genotypes, (2) genetic structure of Frankia might be differentiated in small spatial scales, and (3) the different communities of mutualistic partners might be constructed among host ages.

Assembly of bacterial symbionts for rhizosphere soil under host plants

Numerous Frankia ASVs were detected from both rhizosphere and riparian soils while the number of ASVs from root nodules was limited (Fig. S1). Frankia communities were more diverse in the rhizosphere than in the communities in nodules and in riparian soils even when Frankia sequences were separated by OTUs (Fig. 1). This finding indicated that phylogenetically distant genotypes were assembled in host rhizosphere soil.

The higher Frankia diversity in rhizosphere soils compared to riparian soils can be explained by the occurrence of hosts. In rhizosphere soil, free-living Frankia obtains carbon sources from plant root exudates (Chaia et al., 2010; Ronkko et al., 1993; Samant et al., 2015; 2016; Smolander et al., 1990; Valdes, 2008). In addition, secondary metabolites, such as flavonoids, in root exudates act as signals during the development of plantmicrobe symbiosis associations, including actinorhizal symbioses (Hughes et al., 1999; Perrine-Walker et al., 2011; Steinkellner et al., 2007). These chemical compounds in the host's root exudates may promote the local assembly of Frankia in the host's rhizosphere, regardless of infective or non-infective types. Also, the genetic community compositions of Frankia in the host's rhizosphere were affected by the soil environment (Table 1). This result suggests that conspecific host individuals might be exposed to different genotypes of bacterial symbionts in natural environments. Soil conditions, such as soil $\mathrm{pH}$ and nutrients, are important drivers to shape rhizospheric microbial community structure in nature (Fierer et al., 2012; Wei et al., 2017). However, the knowledge of rhizobial communities in soil, including non-infective strains, is still limited. Future studies should pay attention to the assembly processes of rhizobial communities in soil to understand how interacting bacterial members are determined in a natural ecosystem.

\section{Genetic variation in filtering force of mutualistic partnerships}

Despite symbiosis filtering to favor specific genotypes, genetic diversity of Frankia was high in the rhizosphere soil under a host plant. Consistently, previous studies have found that genetic variation in mutualistic partners is sustained in nature (Barrett et al, 2015; 2016; Clawson et al., 1998; 1999; Huguet et al., 2001; Polme et al., 2014). However, stabilizing mechanisms alone cannot explain the persistence of the variation because the evolutionary fixation of the most beneficial partner genotypes is predictable (Heath \& Stinchcombe, 2014; Law \& Koptur, 1986; Parker, 1999; Weyl et al., 2010). Furthermore, the fixation of the beneficial genotypes may lead to the loss of discrimination ability by hosts if it needs a cost (Foster \& Kokko, 2006; Frederickson, 2013; Heath \& Tiffin, 2009; McNamara \& Leimar, 2010). One solution to this paradox is mutation-selection balance (Heath \& Stinchcombe, 2014): the hypothesis that variation in partner quality is maintained by the balance in mutation, which introduces genotypes of lower quality, and selection, which acts to favor the partner genotypes of higher quality. Genetically diverse pool and strong filtering for symbiosis found in this study supports the mutation-selection balance hypothesis. This study suggests mutation-selection balance in the field. Future studies should pay attention to quantifying the strength of selection that stabilizing 
mechanisms impose on partner quality in nature. This would provide great insights for an understanding of the evolution of partner quality and the maintenance of genetic diversity in symbiosis (Heath \& Stinchcombe, 2014).

This study quantified the symbiosis filtering force using a community dissimilarity approach and found the possibility of genetic variation of hosts in the filtering forces in the field (Fig. 3). The reason why similar bacterial compositions were sustained among almost all alders in spite of the variation in the filtering force among host individuals is likely to be explained by the spatial heterogeneity in genetic diversity of Frankia . Frankia genotypes are heterogeneously dispersed in forests (Kagiya and Utsumi 2020). Additionally, NMDS revealed the high variation of Frankia composition in rhizosphere soils among host individuals (Fig. 2a). The necessity of filtering force could depend on genetic diversity and/or the relative abundance ofFrankia genotypes in the surrounding local soil. In fact, the genetic community structure of bacterial symbionts in the host's rhizosphere soil differed in line with soil nutrients, whereas no effects of genetic variation in a host plant were detected to shape rhizospheric bacterial communities (Table 1). If the ability of filtering requires costs and a common optimal symbiont composition is consistent among host individuals, the balance between the necessity and costs can maintain the homogeneity of symbiont composition in a host plant along with genetic variation in filtering force.

The variation in filtering force might also contribute to the maintenance of genetic variation in microsymbionts. When variation in stabilizing mechanisms alters selection pressure for microsymbionts, genetic variation in microsymbionts, even those involving lower quality genotypes, may be sustained (Heath \& Stinchcombe, 2014). For example, some studies have reported that sanctions are not perfectly effective because they cannot respond to non-zero benefit levels or cannot punish individual partner genotypes (Charlotte et al., 2012; Kiers et al., 2006). If genetic variation in a host plant alters the strength of symbiosis filtering, genetic variation in the host will be the key to revealing coevolution in mutualistic interactions under natural ecosystems with a high genetic diversity of microsymbionts.

The present study clearly demonstrates that host plants interact with specific symbiont genotypes via symbiosis filtering in the field. Our finding that symbiosis filtering indices correlated with the genetic distance of hosts is likely to be evidence of linkage between host genetics and symbiont assembly in nature, even if symbiosis filtering indices depend on the dissimilarity of Frankia composition in rhizosphere soils among rivers and/or non-infective strains that make upFrankia communities in rhizosphere soils. Toward an integrative understanding of mutualistic coevolution and community assembly in natural ecosystems, future studies should pay attention to differences in partner quality and infectivity between infecting and non-infecting members of communities. Moreover, revealing the genetic basis underlying stabilizing mechanisms is fruitful toward an understanding of the interplay between coevolutionary dynamics in mutualism and community assembly in symbiosis.

\section{Acknowledgement}

We thank Takashi Saitoh, Osamu Kishida, and Shiro Tsuyuzaki for their helpful and insightful comments on the manuscript. We also thank Rei Sakai, Michio Ishihara, Yoshiaki Takahashi, Masayuki Watarai, Wataru Mamiya, Toru Miyazaki, and members of Uryu Experimental Forest, Hokkaido University, for their field assistance. This research was supported by JSPS KAKENHI Grant Numbers 16J03194 to SK, 16H06179 to SU, and $19 \mathrm{H} 02974$ to SU.

\section{Author contribution}

SK and SU designed and conducted the investigation, performed the molecular analysis, and analyzed the data. SK, KK, and SU wrote the manuscript.

\section{Data availability statement}

The raw metabarcoding data are deposited at DRA with accession numbers of DRA011797 (submission), PRJDB11469 (Bioproject), SAMD00293210-SAMD00293347 (Biosample), DRX275352-DRX275489 (Experiment), and DRR285895-DRR286032 (Run). 


\section{Reference}

Anderson MD, Ruess RW, Myrold DD, Taylor DL (2009) Host species and habitat affect nodulation by specific Frankia genotypes in two species of Alnus in interior Alaska. Oecologia, 160, 619-630. doi:10.1007/s00442-009-1330-0 Archetti M, Scheuring I (2013) Trading public goods stabilizes interspecific mutualism. Journal of Theoretical Biology, 318, 58-67. doi:10.1016/j.jtbi.2012.10.022 Archetti M, Ubeda F, Fudenberg D, Green J, Pierce NE, Yu DW (2011) Let the right one in: a microeconomic approach to partner choice in mutualisms. The American Naturalist, 177, 75-85. doi:10.1086/657622 Bailey J, Hendry A, Kinnison M et al. (2009). From genes to ecosystems: an emerging synthesis of eco-evolutionary dynamics. New Phytologist, 184, 746-749. http://www.jstor.org/stable/27735828 Bangert RK, Turek RJ, Rehill B et al. (2006) A genetic similarity rule determines arthropod community structure. Molecular Ecology, 15, 1379-1391. doi: 10.1111/j.1365-294X.2005.02749.x Barrett LG, Bever JD, Bissett A, Thrall PH (2015) Partner diversity and identity impacts on plant productivity in Acacia-rhizobial interactions. Journal of Ecology, 103, 130-142. doi:10.1111/1365-2745.12336 Barrett LG, Zee PC, Bever JD, Miller JT, Thrall PH (2016) Evolutionary history shapes patterns of mutualistic benefit inAcacia-rhizobial interactions. Evolution, 70, 1473-1485. doi:10.1111/evo.12966 Bates D, Maechler M, Bolker B, Walker S, Grothendieck G, Green P, Fox J (2019) Package "Ime4." October, pp. 1-6. Ben Tekaya S, Guerra T, Rodriguez D, Dawson JO, Hahn D (2018) Frankia diversity in host plant root nodules is independent of abundance or relative diversity of Frankia populations in corresponding rhizosphere soils. Applied and Environmental Microbiology, 84, 1-11. doi:10.1128/AEM.02248-17 Benson DR, Dawson JO (2007) Recent advances in the biogeography and genecology of symbiotic Frankia and its host plants. Physiologia Plantarum, 130, 318-330. doi:10.1111/j.13993054.2007.00934.x Berry AM, Mendoza-Herrera A, Guo Y, et al. (2011) New perspectives on nodule nitrogen assimilation in actinorhizal symbioses. Functional Plant Biology, 38, 645. doi:10.1071/FP11095 Bittebiere AK, Benot ML, Mony C (2020) Clonality as a key but overlooked driver of biotic interactions in plants. Perspectives in Plant Ecology, Evolution and Systematics, 43, 1433-8319, doi:10.1016/j.ppees.2020.125510. Blois JL, Williams JW, Fitzpatrick MC et al. (2013) Modeling the climatic drivers of spatial patterns in vegetation composition since the Last Glacial Maximum. Ecography, 36, 460-473. doi:10.1111/j.1600-0587.2012.07852.x Bolyen E, Rideout JR, Dillon MR et al. (2019) Reproducible, interactive, scalable and extensible microbiome data science using QIIME 2. Nature Biotechnology, 37, 852-857. doi:10.1038/s41587-019-0209-9 Broughton WJ, Zhang F, Perret X et al. (2003) Signals exchanged between legumes and Rhizobium: agricultural uses and perspectives. Plant and Soil, 252, 129-137. doi:10.1023/A:1024179717780 Callahan BJ, McMurdie PJ, Rosen MJ, Han AW, Johnson AJA, Holmes SP (2016) DADA2: High-resolution sample inference from Illumina amplicon data. Nature Methods, 13, 581-583. doi:10.1038/nmeth.3869 Camacho C, Coulouris G, Avagyan V, Ma N, Papadopoulos J, Bealer K, Madden TL (2009) BLAST+: architecture and applications. BMC Bioinformatics, 10, 421. doi:10.1186/1471-2105-10-421 Capinha C, Essl F, Seebens H, Moser D, Pereira HM (2015) The dispersal of alien species redefines biogeography in the Anthropocene. Science, 348, 12481251. doi:10.1126/science.aaa8913 Chaia EE, Wall LG, Huss-Danell K (2010) Life in soil by the actinorhizal root nodule endophyte Frankia. A review. Symbiosis, 51, 201-226. doi:10.1007/s13199-010-0086-y Chao A, Jost L (2012) Coverage-based rarefaction and extrapolation: standardizing samples by completeness rather than size. Ecology, 93, 2533-2547. doi:10.1890/11-1952.1 Charlotte Jander K, Herre EA, Simms EL (2012) Precision of host sanctions in the fig tree-fig wasp mutualism: consequences for uncooperative symbionts. Ecology Letters, 15, 1362-1369. doi:10.1111/j.1461-0248.2012.01857.x

Chen Z, Li J (2004) Phylogenetics and biogeography of Alnus (Betulaceae) inferred from sequences of nuclear ribosomal DNA ITS region. Int J Plant Sci, 165:325-335. doi:10.1086/382795

Clawson ML, Caru M, Benson DR (1998) Diversity of Frankia strains in root nodules of plants from the families Elaeagnaceae and Rhamnaceae. Applied and Environmental Microbiology, 64, 3539-3543. doi:10.5301/HIP.2012.9281 Clawson, Michael L, Gawronski J, Benson DR (1999) Dominance ofFrankia strains in stands of Alnus incana subsp.rugosa and Myrica pensylvanica. Canadian Journal of Botany, 77, 1203-1207. Crutsinger GM, Collins MD, Fordyce JA, Gompert Z, Nice CC, Sanders NJ (2006) Plant genotypic diversity predicts community structure and governs an ecosystem process. Science, 313, 966-968. doi:10.1126/science.1128326 Crutsinger GM, Rodriguez-Cabal MA, Roddy AB et al. (2014). Genetic vari- 
ation within a dominant shrub structures green and brown community assemblages. Ecology, 95, 387-398. doi:10.1890/13-0316.1 de Souza TAF, Freitas H (2017) Arbuscular mycorrhizal fungal community assembly in the Brazilian tropical seasonal dry forest. Ecological Processes, 6, 2. doi:10.1186/s13717-017-0072-x Dean JM, Mescher MC, de Moraes CM (2009) Plant-rhizobia mutualism influences aphid abundance on soybean. Plant and Soil, 323, 187-196. doi:10.1007/s11104-009-9924-1 Denison RF. (2000). Legume sanctions and the evolution of symbiotic cooperation by rhizobia. The American Naturalist, 156, 567. doi:10.2307/3079063 Dungey HS, Potts BM, Whitham TG, Li HF (2000) Plant genetics affects arthropod community richness and composition: Evidence from a synthetic eucalypt hybrid population. Evolution, 54, 1938-1946. doi:10.1111/j.0014-3820.2000.tb01238.x Ehrlich PR, Raven PH (1964) Butterflies and plants: a study in coevolution. Evolution, 18, 586. doi:10.2307/2406212 Ellison AM, Bank MS, Clinton BD et al. (2005) Loss of foundation species: consequences for the structure and dynamics of forested ecosystems. Frontiers in Ecology and the Environment, 3, 479-486. doi:10.1890/1540-9295(2005)003[0479:LOFSCF]2.0.CO;2 Ellison AM, Barker-Plotkin AA, Foster DR, Orwig DA (2010) Experimentally testing the role of foundation species in forests: the Harvard Forest Hemlock Removal Experiment. Methods in Ecology and Evolution, 1, 168-179. doi:10.1111/j.2041-210x.2010.00025.x Endara M, Coley PD, Ghabash G et al. (2017) Coevolutionary arms race versus host defense chase in a tropical herbivore-plant system. Proceedings of the National Academy of Sciences, 114: 7499-7505. doi:10.1073/pnas.1707727114 Evans JA, Lankau RA, Davis AS, Raghu S, Landis DA (2016) Soil-mediated eco-evolutionary feedbacks in the invasive plant Alliaria petiolata. Functional Ecology, 30, 1053-1061. doi:10.1111/1365-2435.12685 Evans LM, Clark JS, Whipple AV, Whitham TG (2012) The relative influences of host plant genotype and yearly abiotic variability in determining herbivore abundance. Oecologia, 168, 483-489. doi:10.1007/s00442-011-2108-8 Ferrier S, Manion G, Elith J, Richardson K (2007) Using generalized dissimilarity modelling to analyse and predict patterns of beta diversity in regional biodiversity assessment. Diversity and Distributions, 13, 252-264. doi:10.1111/j.1472-4642.2007.00341.x Fitzpatrick MC, Sanders NJ, Normand S, Svenning J, Ferrier S, Gove AD, Dunn RR (2013) Environmental and historical imprints on beta diversity: insights from variation in rates of species turnover along gradients. Proceedings of the Royal Society B: Biological Sciences, 280, 20131201. doi:10.1098/rspb.2013.1201 Floate KD, Martinsen GD, Whitham TG (1997) Cottonwood hybrid zones as centres of abundance for gall aphids in western North America: importance of relative habitat size. The Journal of Animal Ecology, 66), 179. doi:10.2307/6020 Foster KR, Kokko H (2006) Cheating can stabilize cooperation in mutualisms. Proceedings of the Royal Society B: Biological Sciences, 273, 2233-2239. doi:10.1098/rspb.2006.3571 Frederickson ME (2013) Rethinking mutualism stability: cheaters and the evolution of sanctions. The Quarterly Review of Biology, 88, 269-295. doi:10.1086/673757 Fritz RS, Gaud WS, Sacchi CF, Price PW (1987) Variation in herbivore density among host plants and its consequences for community structure. Oecologia, 72, 577-588. doi:10.1007/BF00378986 Fukuzawa K, Shibata H, Takagi K et al. (2006) Effects of clear-cutting on nitrogen leaching and fine root dynamics in a cool-temperate forested watershed in northern Japan. Forest Ecology and Management, 225, 257-261. doi:10.1016/j.foreco.2006.01.001 Futuyma DJ, Agrawal AA (2009) Macroevolution and the biological diversity of plants and herbivores. Proceedings of the National Academy of Sciences, 106, 18054-18061. doi:10.1073/pnas.0904106106 Genung MA, Bailey JK, Schweitzer JA (2013) Belowground interactions shift the relative importance of direct and indirect genetic effects. Ecology and Evolution, 3, 1692-1701. doi:10.1002/ece3.582 He XH, Chen LG, $\mathrm{Hu}$ XQ, Asghar S (2004) Natural diversity of nodular microsymbionts of Myrica rubra. Plant and Soil, 262, 229-239. doi:10.1023/B:PLSO.0000037045.42440.1d Heath KD, Stinchcombe JR (2014) Explaining mutualism variation: a new evolutionary paradox? Evolution, 68, 309-317. doi:10.1111/evo.12292 Heath KD, Tiffin P (2009) Stabilizing mechanisms in a legume-Rhizobium mutualism. Evolution, 63, 652-662. doi:10.1111/j.1558-5646.2008.00582.x Hoeksema JD, Kummel M (2003) Ecological persistence of the plantmycorrhizal mutualism: a hypothesis from species coexistence theory. The American Naturalist, 162, S40S50. doi:10.1086/378644 Hughes M, Donnelly C, Crozier A, Wheeler CT (1999) Effects of the exposure of roots of Alnus glutinosa to light on flavonoids and nodulation. Canadian Journal of Botany, 77, 1311-1315. doi:10.1139/cjb-77-9-1311 Huguet V, Batzli JM, Zimpfer JF, Normand P, Dawson JO, Fernandez MP (2001) Diversity and Specificity of Frankia Strains in Nodules of Sympatric Myrica gale, Alnus incana, and Shepherdia canadensis Determined by rrs Gene Polymorphism. Applied and Environmental Microbiology, 67, 
2116-2122. doi:10.1128/AEM.67.5.2116-2122.2001 Johnson MTJ, Lajeunesse MJ, Agrawal AA (2006) Additive and interactive effects of plant genotypic diversity on arthropod communities and plant fitness. Ecology Letters, 9, 24-34. doi:10.1111/j.1461-0248.2005.00833.x Kagiya S, Yasugi M, Kudoh H, Nagano AJ, Utsumi S (2018) Does genomic variation in a foundation species predict arthropod community structure in a riparian forest? Molecular Ecology, 27, 1284-1295. doi:10.1111/mec.14515 Kiers ET, Denison RF (2008) Sanctions, cooperation, and the stability of plant-rhizosphere mutualisms. Annual Review of Ecology, Evolution, and Systematics, 39, 215-236. doi:10.1146/annurev.ecolsys.39.110707.173423 Kiers ET, Hutton MG, Denison RF (2007) Human selection and the relaxation of legume defences against ineffective rhizobia. Proceedings of the Royal Society B: Biological Sciences, 274, 3119-3126. doi:10.1098/rspb.2007.1187 Kiers ET, Rousseau RA, Denison RF (2006) Measured sanctions: Legume hosts detect quantitative variation in rhizobium cooperation and punish accordingly. Evolutionary Ecology Research, 8, 1077-1086. Kiers ET, Rousseau RA, West SA, Denison RF (2003) Host sanctions and the legume-Rhizobium mutualism. Nature, 425, 78-81. Kimura M (1980). A simple method for estimating evolutionary rates of base substitutions through comparative studies of nucleotide sequences. Journal of Molecular Evolution, 16, 111-120. doi:10.1007/BF01731581 Kumar S, Stecher G, Tamura K (2016) MEGA7: Molecular Evolutionary Genetics Analysis Version 7.0 for Bigger Datasets. Molecular Biology and Evolution, 33, 1870-1874.doi:10.1093/molbev/msw054 Lamit LJ, Busby PE, Lau MK et al. (2015) Tree genotype mediates covariance among communities from microbes to lichens and arthropods. Journal of Ecology, 103, 840-850. doi:10.1111/1365-2745.12416 Lankau RA (2011) Intraspecific variation in allelochemistry determines an invasive species' impact on soil microbial communities. Oecologia, 165, 453-463. doi:10.1007/s00442-010-1736-8 Lasram FBR, Hattab T, Halouani G, Romdhane MS, Le Loc'h F (2015) Modeling of beta diversity in tunisian waters: predictions using generalized dissimilarity modeling and bioregionalisation using fuzzy clustering. PLOS ONE, 10, e0131728. doi:10.1371/journal.pone.0131728 Law R, Koptur S (1986) On the evolution of non-specific mutualism. Biological Journal of the Linnean Society, 27, 251-267. doi:10.1111/j.1095-8312.1986.tb01736.x Lekberg Y, Meadow J, Rohr JR, Redecker D and Zabinski CA (2011) Importance of dispersal and thermal environment for mycorrhizal communities: lessons from Yellowstone National Park. Ecology, 92, 1292-1302. doi:10.1890/10-1516.1 Li W, Godzik A (2006) Cd-hit: a fast program for clustering and comparing large sets of protein or nucleotide sequences. Bioinformatics, 22, 1658-1659. doi:10.1093/bioinformatics/btl158 Lopez-Garcia A, Varela-Cervero S, Vasar M, Opik M, Barea JM, Azcon-Aguilar C (2017) Plant traits determine the phylogenetic structure of arbuscular mycorrhizal fungal communities. Molecular Ecology, 26, 6948-6959. doi:10.1111/mec.14403 Materon LA, Zibilske L (2001) Delayed inoculation and competition of nitrogen-fixing strains in Medicago noeana (Boiss.) andMedicago polymorpha (L.). Applied Soil Ecology, 17, 175-181. doi:10.1016/S0929-1393(01)00121-4 McNamara JM, Leimar O (2010) Variation and the response to variation as a basis for successful cooperation. Philosophical Transactions of the Royal Society B: Biological Sciences, 365, 2627-2633. doi:10.1098/rstb.2010.0159 Mensah JA, Koch AM, Antunes PM et al. (2015) High functional diversity within species of arbuscular mycorrhizal fungi is associated with differences in phosphate and nitrogen uptake and fungal phosphate metabolism. Mycorrhiza, 25, 533-546. doi:10.1007/s00572015-0631-x Mishra AK, Singh PK, Singh P et al. (2015) Phylogeny and evolutionary genetics of Frankia strains based on 16S rRNA and nifD-K gene sequences. Journal of Basic Microbiology, 55, 1013-1020. doi:10.1002/jobm.201400914 Moreira X, Mooney KA (2013) Influence of plant genetic diversity on interactions between higher trophic levels. Biology Letters, 9, 20130133. doi:10.1098/rsbl.2013.0133 Nei M (1978) Estimation of average heterozygosity and genetic distance from a small number of individuals. Genetics, 89, 583-590. Retrieved from https://www.genetics.org/content/89/3/583.article-info Normand P, Lapierre P, Tisa LS et al. (2007) Genome characteristics of facultatively symbiotic Frankia sp. strains reflect host range and host plant biogeography. Genome Research, 17, 7-15. doi:10.1101/gr.5798407 Nouioui I, Cortesalbayay C, Carro L et al. (2019) Genomic insights into plant-growth-promoting potentialities of the genus Frankia. Frontiers in Microbiology, 10, 1-15. doi:10.3389/fmicb.2019.01457 Nouioui I, Ghodhbane-Gtari F, Fernandez MP et al. (2014) Absence of cospeciation between the uncultured Frankia microsymbionts and the disjunct actinorhizal Coriaria species. BioMed Research International, 2014. doi:10.1155/2014/924235 Parker MA (1999) Mutualism in metapopulations of legumes and rhizobia. American Naturalist, 153, 4860. doi:10.1086/303211 Peret B, Swarup R, Jansen L et al. (2007) Auxin influx activity is associated with 
Frankia infection during actinorhizal nodule formation in Casuarina glauca. Plant Physiology, 144, 18521862. doi:10.1104/pp.107.101337 Perrine-Walker F, Gherbi H, Imanishi L et al. (2011) Symbiotic signaling in actinorhizal symbioses. Current Protein \& Peptide Science, 999, 1-9. doi:10.2174/1389211213488422037 Polme S, Bahram M, Koljalg U, Tedersoo L (2014) Global biogeography of Alnus-associated Frankia actinobacteria. New Phytologist, 204, 979-988. doi:10.1111/nph.12962

Ren BQ, Xiang XG, Chen ZD (2010) Species identification of Alnus (Betulaceae) using nrDNA and cpDNA genetic markers. Mol Ecol Resour 10, 594-605. doi:10.1111/j.1755-0998.2009.02815.x

Rodriguez D, Guerra TM, Forstner MRJ, Hahn D (2016) Diversity ofFrankia in soil assessed by Illumina sequencing of nifH gene fragments. Systematic and Applied Microbiology, 39, 391-397. doi:10.1016/j.syapm.2016.06.007 Ronkko R, Smolander A, Nurmiaho-Lassila EL, Haahtela K (1993)Frankia in the rhizosphere of nonhost plants: A comparison with root-associated N2-fixing Enterobacter, Klebsiella andPseudomonas. Plant and Soil, 153, 85-95. doi:10.1007/BF00010547 Samant S, Dawson JO, Hahn D (2015). Growth responses of indigenousFrankia populations to edaphic factors in actinorhizal rhizospheres. Systematic and Applied Microbiology, 38, 501-505. doi:10.1016/j.syapm.2015.07.005 Samant S, Dawson JO, Hahn D (2016) Growth responses of introducedFrankia strains to edaphic factors. Plant and Soil, 400, 123-132. doi:10.1007/s11104-015-2720-1 Sayed WF, El-Sharouny HM, Zahran HH, Ali WM (2002) Composition ofCasuarina leaf litter and its influence onFrankia-Casuarina symbiosis in soil. Folia Microbiologica, 47, 429-434. doi:10.1007/BF02818703 Schubert KR (1986) Products of biological nitrogen fixation in higher plants: synthesis, transport, and metabolism. Annual Review of Plant Physiology, 37, 539-574. doi:10.1146/annurev.pp.37.060186.002543 Schweitzer JA, Juric I, van de Voorde TFJ, Clay K, van der Putten WH, Bailey JK (2014) Are there evolutionary consequences of plant-soil feedbacks along soil gradients? Functional Ecology, 28, 55-64. doi:10.1111/1365-2435.12201 Schweitzer JA, Madritch MD, Bailey JK et al. (2008) From genes to ecosystems: the genetic basis of condensed tannins and their role in nutrient regulation in a populus model system. Ecosystems, 11, 1005-1020. doi:10.1007/s10021-008-9173-9 Schweitzer JA, Van Nuland M, Bailey JK (2018) Intraspecific plant-soil feedbacks link ecosystem ecology and evolutionary biology. In Ohgushi T, Wurst S, Johnson SN (Eds.), Aboveground-Belowground Community Ecology, 69-84. doi:10.1007/978-3-319-91614-9_4 Smolander A, Ronkko R, Nurmiaho-Lassila E, Haahtela K (1990) Growth ofFrankia in the rhizosphere of Betula pendula, a nonhost tree species. Canadian Journal of Microbiology, 36, 649-656. doi:10.1139/m90-111 Steinkellner S, Lendzemo V, Langer I, Schweiger P, Khaosaad T, Toussaint J, Vierheilig H (2007) Flavonoids and strigolactones in root exudates as signals in symbiotic and pathogenic plant-fungus interactions. Molecules, 12, 1290-1306. doi:10.3390/12071290 terHorst CP, Zee PC (2016) Eco-evolutionary dynamics in plant-soil feedbacks. Functional Ecology, 30, 1062-1072. doi:10.1111/13652435.12671 Tisa LS, Oshone R, Sarkar I, Ktari A, Sen A, Gtari M (2016) Genomic approaches toward understanding the actinorhizal symbiosis: an update on the status of the Frankia genomes. Symbiosis, 70, 5-16. doi:10.1007/s13199-016-0390-2 Turelli M (1985) Effects of pleiotropy on predictions concerning mutation-selection balance for polygenic traits. Genetics, 111, 165-195. Utsumi S, Ando Y, Craig TP, Ohgushi T (2011) Plant genotypic diversity increases population size of a herbivorous insect. Proceedings of the Royal Society B: Biological Sciences, 278, 3108-3115. doi:10.1098/rspb.2011.0239 Valdes M (2008) Frankia Ecology. In Pawlowski K, Newton WE (Eds.), Nitrogen-fixing Actinorhizal Symbioses, 49-71. Dordrecht, The Netherlands: Springer US. West SA, Kiers ET, Pen I, Denison RF (2002a). Sanctions and mutualism stability: when should less beneficial mutualists be tolerated? Journal of Evolutionary Biology, 15, 830-837. doi:10.1046/j.1420-9101.2002.00441.x West SA, Kiers ET, Simms EL, Denison RF (2002b). Sanctions and mutualism stability: why do rhizobia fix nitrogen? Proceedings of the Royal Society of London. Series B: Biological Sciences, 269, 685-694. doi:10.1098/rspb.2001.1878 Weyl EG, Frederickson ME, Yu DW, Pierce NE (2010) Economic contract theory tests models of mutualism. Proceedings of the National Academy of Sciences of the United States of America, 107, 15712-15716. doi:10.1073/pnas.1005294107 Whitham TG, Bailey JK, Schweitzer JA et al. (2006) A framework for community and ecosystem genetics: from genes to ecosystems. Nature Reviews Genetics, 7, 510-523. doi:10.1038/nrg1877 Whitham TG, Morrow PA, Potts BM (1994) Plant hybrid zones as centers of biodiversity: the herbivore community of two endemic Tasmanian eucalypts. Oecologia, 97, 481-490. doi:10.1007/BF00325886 Wimp GM, Martinsen GD, Floate 
KD, Bangert RK, Whitham TG (2005) Plant genetic determinants of arthropod community structure and diversity. Evolution, 59, 61-69. doi:10.1111/j.0014-3820.2005.tb00894.x

\section{Figure legends}

Fig. 1. Bipartite incidence networks of Frankia OTUs withinAlnus host individuals (a) and soils (b). Samples and detectedFrankia OTUs from each sample are represented x- and y-axes, respectively. (b) gray area represents OTUs from riparian soils and white area represents OTUs from host rhizosphere soils. Colors indicates each river area (blue: BT; red: DR; green: SE; yellow: UT).

Fig. 2. (a) Non-metric multidimensional scaling of Frankiacommunity among nodules and rhizosphere of the A. hirsuta, and riparian soils. Circles indicated Frankia communities in hosts' root-nodules. Triangles indicated the communities in rhizosphere soils. Squares indicated the communities in riparian soils. (b) FrankiaOTU richness in host's root nodules and null model by random sampling. The asterisk represents P-value of difference of OTU richness between in nodules and null model by GLM (***: $P<0.001$ ).

Fig. 3. (a) Correlation between genetic distance of A. hirsutaand symbiosis filtering difference. (b) Proportions of unique and shared deviance explained on symbiosis filtering difference. GD: Nei's genetic distance of hosts (black); OTHER: measured predictors including spatial distance among hosts, soil pH, amount of inorganic nitrogen in soils, and community dissimilarity of Frankia in rhizosphere soils based on Jaccard index (gray); BOTH: both predictors (white).

\section{Tables}

Table 1. Correlations of Frankia communities in root-nodules and soils with genetic distance of alders and soil conditions.

\begin{tabular}{lllllll}
\hline & Host's genetic distance & Host's genetic distance & Soil pH & Soil pH & Inorganic N & Inorganic $~$ \\
\hline & $r$ & $P$ & $R^{2}$ & $P$ & $R^{2}$ & $P$ \\
Nodule community & -0.309 & 0.903 & 0.153 & 0.477 & 0.307 & 0.189 \\
Rhizosphere community & -0.137 & 0.754 & 0.212 & 0.371 & $\mathbf{0 . 5 8 1}$ & $\mathbf{0 . 0 1 7}$ \\
Riparian community & 0.180 & 0.213 & 0.090 & 0.654 & 0.146 & 0.512 \\
\hline
\end{tabular}



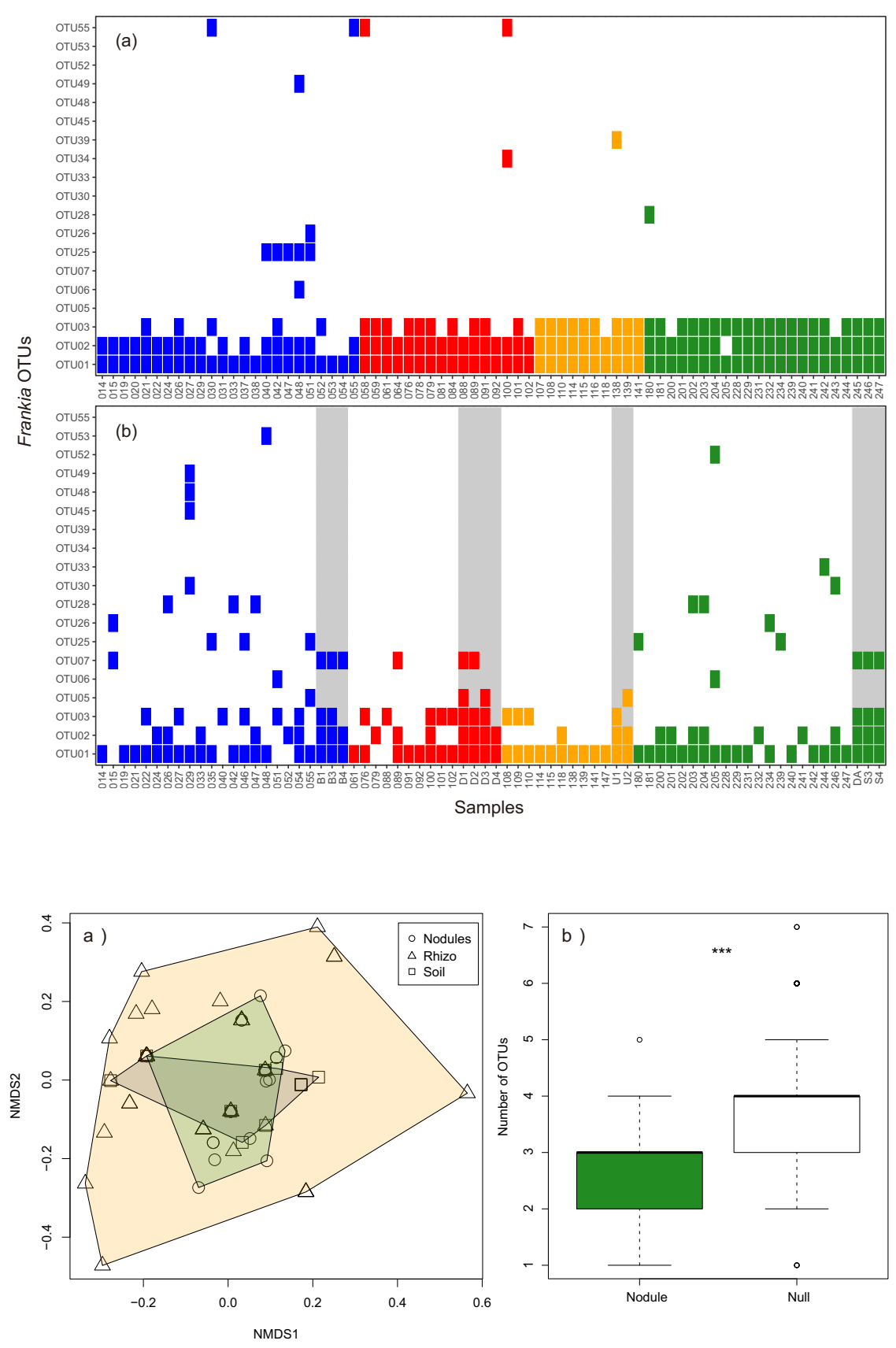
a.

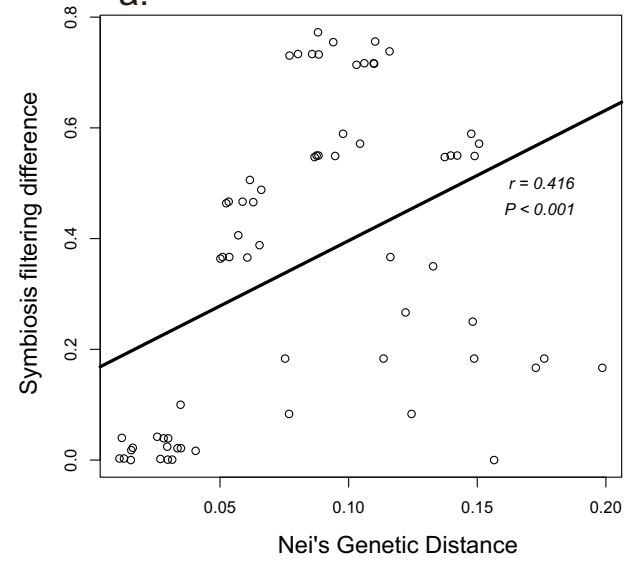

b.

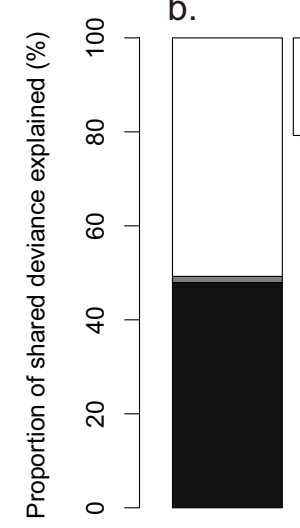

$\begin{array}{ll}\square & \text { BOTH } \\ \square & \text { OTHER }\end{array}$

- GD 